\title{
Klusen und verwandte Formen im Schweizer Jura
}

\begin{abstract}
Kluse and Related Forms in the Swiss Jura Mountains. - It is shown that the usual theory of the genesis of the "kluses" (transverse gorges cutting across ridges) in the Jura mountains of Switzerland by fluvial erosion by antecedent rivers is untenable:

1 st, the erosive power of a river on its bed is by at least a factor 100 too small to abrade solid rock as found in the kluses. $2 n d$, the orientation structure of the segments of the rivers flowing in the kluses is non-random which, by the tenets of the principle of antagonism in landscape development, is indicative of a non-exogenic origin of them.

$3 r d$, the orientation structure of the klus-rivers agrees with that of the joints in the area.

4 th, in view of the fact that the joints are known to have been caused by recent plate-tectonic processes, the same must be assumed for the kluses: the latter owe their genesis to complicated geologic lineaments, folds and shear faults. This fact has practical consequences: During the construction of tunnels underneath a klus one has to take into consideration that the disturbance in the landscape represented by a klus my well reach geologically far into the basement. 5 th, the erosion of the kluses occurred in parallel to the direction of the joints. In this instance, the debris produced by the tectonic processes and by frost action was removed by the mechanical and chemical action of the water. During the cold times and cold spells during warm times this water was mainly melt-water.

6 th, special studies are necessary for the determination of the quantity of debris that was removed. The time-span available for this removal is much longer than commonly assumed: it begins with the first tectonic folding, in the Jura mountains already in mid-Miocene, $15 \mathrm{Ma}$ ago.
\end{abstract}

\section{Einleitung}

Im Schweizer Faltenjura bestimmen generell SW-NE streichende tektonische Strukturen das Landschaftsbild: Die Antiklinalen bilden Gebirgsketten, die Synklinalen Längstäler und Becken. Die Gebirgsketten werden aber immer wieder von markanten, schluchtartigen Formen durchbrochen: von Klusen und verwandten Gebilden. Ihre Bildung ist nach landläufiger Vorstellung durch Antezedenz (= fluviale Eintiefung während der Faltung) entstanden (z. B. LABHART, 1991); demnach "sägte» sich ein schon bestehender Fluß in die sich hebende Antiklinale in dem Maße ein, wie diese sich hob. Noch früher galt die Ansicht, eine Klus wäre durch zwei Bäche gebildet worden, die von einem Antiklinalrücken nach beiden Seiten in der Fallinie abgeflossen wären und sich immer tiefer einschnitten, bis schließlich die Antiklinale durchsägt gewesen wäre. Nach unserer Meinung sind aber beide Ansichten unhaltbar, und hiefür sollen Argumente gebracht werden.

1. Nach einer einfachen hydromechanischen Überlegung ist die Erosionskraft eines Flusses viel zu gering, als daß er sich in festen Fels einsägen könnte. In der Tat ist die hydraulische Schubspannung am Flußbett quantitativ höchstens von der Größenordnung von 100 kPa (MAGILLIGAN, 1992), während die Scherfestigkeit von festem Fels in der Größenordnung von $10 \mathrm{MPa}$ (siehe z. B. SCHEIDEGGER, 1982, p. 188-189), also zwei Größenordnungen höher, liegt. Daher kann sich ein Fluß niemals in festen Fels «einsägen».

2. HANTKE (1978, p. 284; 1991, p. 264) hat schon aufgrund der Phänomenologie bemerkt, daß die Juraklusen ihre Entstehung oft kompliziert verlaufenden Durchscherungen, Knicken in Faltenachsen und Blattverschiebungen, also endogenen und nicht exogenen Vorgängen, verdanken. Diese Beobachtung kann durch eine statistische Analyse der Orientationsstruktur der Flußsegmente erhärtet werden: Nach dem Antagonismusprinzip in der Landschaftsentwicklung produzieren exogene Vorgänge zufällige, endogene Vorgänge, aber systematische Strukturen (SCHEIDEGGER, 1979). Die Datenbasis für eine derartige Analyse wird dadurch geschaffen, daß die Richtungen (dabei wird das Azimut $\mathrm{N}>\mathrm{E}$ der Polaren, d. h. der Normalen zum jeweiligen "Trend", in Grad angegeben, um den Vergleich mit anderen strukturellen Elementen zu erleichtern) von Segmenten konstanter ( 100 m) Länge des Klusflusses notiert werden. Für diese wird eine Orientierungsanalyse vorgenommen und eine statistische Analyse nach KOHLBECK und SCHEIDEGGER (1977) durchgeführt, welche die bevorzugten Richtungen (d. h. die Richtungsmaxima) der vorhandenen Scharen rechnerisch bestimmt. In allen untersuchten Fällen hat sich gezeigt, daß die Richtungsverteilung nicht zufällig ist und daher nicht exogen bedingt sein kann.

3. Es ergibt sich, daß in allen bekannten Fällen die Orientationsstruktur der Flußrichtungen in den Klusen mit jener der Klüfte in der jeweiligen Umgebung nahezu über-

René Hantke, Prof. Dr., Glärnischstraße 3, 8712 Stäfa A. E. Scheidegger, Prof. Dr., Technische Universität, Abteilung Geophysik, A-1040 Wien 


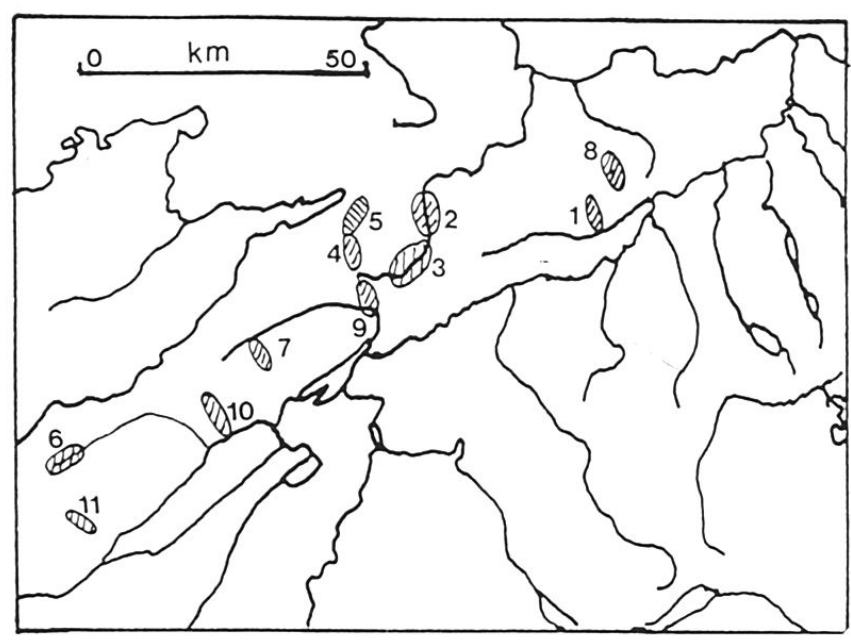

Abb.1 Kartenskizze zur Lage der untersuchten Klusen und Combes. 1: Balsthal-Mümliswil, 2: Moutier-Courrendlin, 3: Court-Moutier, 4: Undervelier-Süd, 5: Undervelier-Nord, 6: St-Sulpice, 7: Combe Grède, 8: Oberer Hauenstein, 9: Sonceboz-Tavannes, 10: Gorges du Seyon, 11: Gorges de Covatanne.

einstimmt. Die Orientationen der Klüfte können ähnlich wie die der Flußsegmente analysiert werden: Fallrichtung und Fallwinkel von Klüften werden gemessen; dann wird nach KOHLBECK und SCHEIDEGGER wieder eine statistische Analyse durchgeführt. Die Notierung für die Kluftwerte ist dabei die standardmäßige von KOHLBECK und SCHEIDEgGer: Aufgelistet sind Azimut $\mathrm{N}>\mathrm{E}$ der Fallrichtung (d. h. der Normalenzur Streichrichtung) und Fallwinkel der bevorzugten Kluftstellungen in Grad (mit Fehlergrenzen); dabei könnten a priori 1, 2, 3 oder 4 Verteilungen (mit entsprechenden Richtungs-«Maxima») vorausgesetzt werden; es stellte sich aber heraus, daß nur Rechnungen mit drei a priori angenommenen Verteilungen sinnvoll sind: bei geringerer Anzahl werden wirklich bestehende Verteilungen gewaltsam in diese vermischt; bei vier Verteilungen hat die vierte entweder kein statistisches Gewicht oder unannehmbare Fehlergrenzen. In den Tabellen sind die (relativen) Gewichte der einzelnen Verteilungen und die Streuung der dazugehörigen Meßwerte angegeben. Die Resultate können dann mit denen der Analyse der Flußsegmente, die gleich wie die der Klüfte (abgesehen von den Fallwinkeln, die hierfür keine Bedeutung haben) in der Tabelle nach der dargelegten Konvention dargestellt sind, verglichen werden: Die Diskrepanzen sind jeweils (in Grad) angegeben. Da die Klüfte durch plattentektonische Prozesse, wohl als Scherflächen, erzeugt wurden (SCHEIDEGGER, 1985), weist die Übereinstimmung zwischen Fluß- und Kluftrichtungen ebenfalls auf einen plattentektonischen Ursprung der Klusen hin.

Diese Thesen sollen anhand von spezifischen Objekten erhärtet werden. Dabei betrachten wir elf Beispiele von Klusen und verwandten Gebilden: Zunächst "klassische» Klusen, die einen Höhenzug durchbrechen, wie die sym- metrischen von Balsthal-Mümliswil, Moutier-Courrendlin, Court-Moutier und Undervelier, sowie die asymmetrische von St-Sulpice; in die "klassische» Kategorie gehören auch steile, seitliche Klusen, so die Combe Grede in der Nordflanke des Chasseral. Dann folgen klusähnliche Übergänge, wie jene am Oberen Hauenstein und bei Sonceboz-Tavannes und schließlich klusartige Schluchten, die eine Randschwelle von einem Hochtal zu einem niedrigeren Niveau durchqueren (Randschwellenschluchten), wie die Gorges du Seyon nördlich von Neuchâtel und jene von Covatanne südlich von Ste-Croix. Abb. 1 zeigt die Orte der elf untersuchten Objekte.

\section{Klassische Klusen}

\section{Klus von Balsthal-Mümliswil}

Als erstes betrachten wir sogenannte «klassische» Klusen: meist symmetrische Gebilde, die an zwei Engstellen, am Anfang und Ende, einen Jurahöhenzug durchbrechen und im Inneren ausgeweitet sind. Als Musterbeispiel möge die Klus von Balsthal-Mümliswil dienen (Abb. 2). In dieser Klus wurden an drei Stellen Kluftorientierungen gemessen, an beiden Enden der Klus in Kalksteinen des Mittleren Malms. Die numerischen Resultate der Auswertung sind in Tabelle 1 aufgelistet.

Dann wurden die Flußrichtungen in der eingangs dargelegten Weise statistisch erfaßt. Die Resultate der Auswertung wurden ebenfalls in Tabelle 1 eingetragen.

Zur Interpretation ist zu sagen, daß bei den Flußsegmenten eine Verteilung von sehr geringem Gewicht ist und die beiden anderen sich voneinander in der Richtung nur um $45^{\circ}$ unterscheiden. Dagegen sind die zwei stärksten Kluftmaxima konjugiert und entsprechen ziemlich genau den in der Schweiz prävalenten «europäischen" Orientierungen (SCHEIDEGGER, 1977). Im ganzen kommen die Kluftmaxima ungefähr den Segmentmaxima gleich (maximal $13^{\circ}$ Unterschied).

\section{Klus Moutier-Courrendlin}

Als weitere klassische Klus wurde der von der Birs zwischen Moutier und Courrendlin durchflossene Durchbruch untersucht. Kluftmessungen wurden an insgesamt fünf Aufschlüssen im Malm gemacht. Am oberen (S-) Eingang der Klus unterhalb von Moutier durchbricht die Birs einen Felsriegel. Mittels Visierung wurde festgestellt, daß die beiden Seiten um $7 \mathrm{~m}$ sinistralgegeneinander verschoben sind. Die Resultate der Auswertung sind in Tabelle 1 wiedergegeben. Dann wurden die Flußrichtungen in der üblichen Weise statistisch erfaßt; es ergaben sich die in Tabelle 1 aufgelisteten Resultate.

Bei der Auswertung der Klüfte zeigt sich eine wichtige Streichrichtung von $\mathrm{N} 13^{\circ} \mathrm{W}$ (Maximum 3 in Tabelle 1; Gewicht 0.39). Diese stimmt etwa mit der generellen Klusrichtung überein: eine gerade Verbindung vom An- 


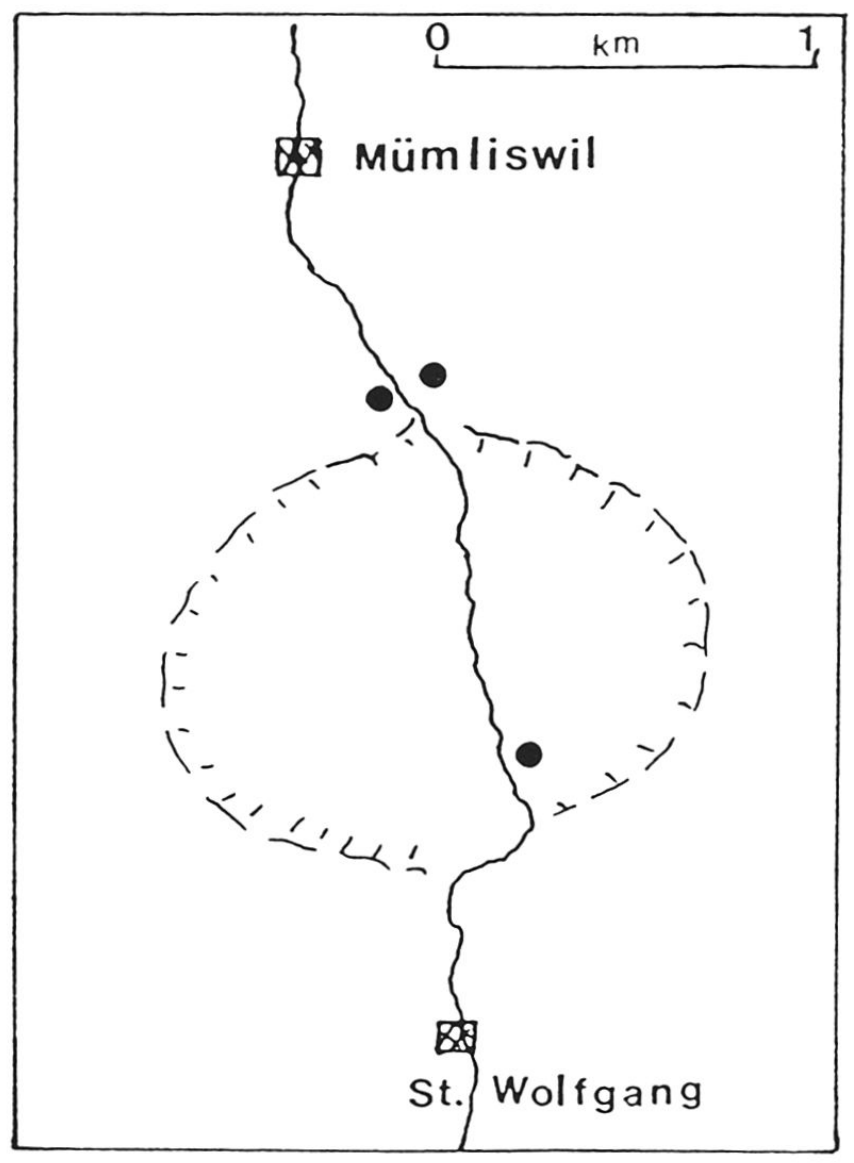

Abb.2 Kartenskizze einer "klassischen" Juraklus: der Klus Balsthal-Mümliswil mit den drei Kluftmeßstellen.

fang der Klus zu ihrem Ende ergibt einen Trend von N $04^{\circ}$ W, d. h. eine Abweichung von der Streichrichtung der entsprechenden Kluftschar von $9^{\circ}$. Sie liegt innerhalb der statistisch ermittelten Fehlergrenzen von $\pm 10^{\circ}$. Die vorliegende Untersuchung zeigt, daß die Schlucht der Richtung einer der Kluftscharen entspricht. Tabelle 1 erlaubt auch eine genauere numerische Analyse: Alle drei Verteilungen bekunden eine $z$. T. sehr gute (Maximum 1 und 2: Diskrepanz $4^{\circ}$ ) oder doch leidliche Entsprechung (Maximum 3: Diskrepanz 19 ${ }^{\circ}$; das dritte Maximum stimmt nur in diesem Rahmen mit der "visuellen» Richtung der Klus überein) zwischen statistisch ermittelten Fluß- und Kluftrichtungen.

Ferner zeigt sich, daß die Kluftrichtungen der untersuchten Klus den «europäischen» plattentektonischen Kluftrichtungen (SCHEIDEGGER, 1977) entsprechen; die «europäische» maximale Druckrichtung (NW-SE) wäre auch für die sinistrale Verschiebung, die am oberen Eingang der Klus festgestellt wurde, verantwortlich. Die These, daß die Klus von Moutier-Courrendlin plattenneotektonisch vorgezeichnet und nicht epigenetisch erzeugt wurde, ist daher voll gestützt.

\section{Klus Court-Moutier}

Oberhalb von Moutier, zwischen Moutier und Court, befindet sich eine weitere klassische Klus. Kluftstellungen wurden an drei Aufschlüssen eingemessen und die Werte statistisch ausgewertet; die Resultate sind in der Tabelle 1 dargestellt. Eine Durchsicht zeigt, daß Maxima bei 193 und $276^{\circ}$ liegen. Die dritte Richtung hat nur ein sehr geringes statistisches Gewicht und scheint unecht zu sein. Die Kluftstellungen in der Klus von Court-Moutier sind kaum verschieden von denen in jener von Moutier-Courrendlin.

Auch die Klus von Court-Moutier wird von der Birs durchflossen; ihr Lauf bot sich für eine Strukturstudie an. Die Resultate sind ebenfalls in Tabelle 1 aufgelistet.

Ein Vergleich der Kluft- mit den Talungsrichtungen ergibt wenigstens im ersten Maximum eine recht gute Übereinstimmung zwischen den beiden. Dies entspricht der Haupttalungsrichtung mit Polaren um $280^{\circ}$. Die zweite Schar (Polare um $161-193^{\circ}$ ) ist jeweils eine konjugierte dazu. Die übrigen Scharen sind eher unbedeutend Die Mittelnormale der beiden Hauptscharen der Klüfte $\left(144^{\circ}\right)$ entspricht wiederum der europäischen plattentektonischen P-(i.e. Druck-)Richtung, was die Theorie bestätigt, daß Klüfte und Klusen durch die rezente Plattentektonik vorgezeichnet wurden und nicht aus Antezedenz eines ehemaligen Flußlaufes hervorgegangen sind.

\section{Klus Undervelier-Süd}

Als weitere klassische Klus wurde jene von UndervelierSüd untersucht, eine der typischen Juraklusen, die von der Sorne von $\mathrm{S}$ nach $\mathrm{N}$ «ausgesägt» worden sein sollen. An vier Aufschlüssen wurden Kluftstellungen eingemessen. Die gemessenen Werte wurden statistisch ausgewertet und die Resultate in der Tabelle 1 aufgeführt. Es zeigen sich drei etwa gleich gewichtige Kluftscharen.

Anschließend wurden die Talrichtungen (Polaren) statistisch analysiert. Die Resultate sind ebenfalls in Tabelle 1 aufgelistet. Wiederum lassen sich drei signifikante Verteilungen erkennen.

Kluft- und Talungsrichtungen stimmen bei allen drei Verteilungen recht gut zusammen; im schlechtesten Fall beträgt die Abweichung $16^{\circ}$, einmal gar nur $7^{\circ}$, so da $\beta$ als erwiesen betrachtet werden kann, daß Klüfte und Talungen denselben genetischen, plattentektonischen Ursprung haben.

\section{Klus Undervelier-Nord}

Flußabwärts an die Klus von Undervelier-Süd schließt jene von Undervelier-Nord an; sie ist gewissermaßen die nördliche Hälfte der "Doppelklus» von Undervelier. Der Name "Doppelklus» trifft allerdings nur bedingt zu, denn es handelt sich um zwei verschiedene Juraketten, die die Sorne von S nach N nacheinander "durchbricht». Dazwischen liegt ein Synklinaltal, aus dem die Sorne kleine Zuflüsse aufnimmt. 
Tabelle 1: Auswertung der Kluft- und Talungsrichtungen

\begin{tabular}{|c|c|c|c|c|c|c|c|}
\hline Balsthal/ZI. & Klüfte & Gew. & Str. & Talungen & Gew. & Str. & Diskr. \\
\hline Max. 1 & $118 \pm 66 / 7443$ & 0.03 & 14 & 120 oder 30009 & 0.22 & 20 & 2 \\
\hline Max. 2 & $35707 / 8614$ & 0.30 & 25 & 9 oder 18908 & 0.00 & 20 & 12 \\
\hline Max. 3 & $8504 / 8512$ & 0.67 & 20 & 72 oder 25200 & 0.78 & 14 & 13 \\
\hline Moutier/ZI. & 106 & & & 69 & & & \\
\hline $\begin{array}{l}\text { Max. } 1 \\
\text { Max. } 2\end{array}$ & $\begin{array}{l}11412 / 8706 \\
18904 / 8800\end{array}$ & $\begin{array}{l}0.29 \\
0.32\end{array}$ & $\begin{array}{l}19 \\
22\end{array}$ & $\begin{array}{r}110 \text { oder } 29009 \\
13 \text { oder } 19300\end{array}$ & $\begin{array}{l}0.53 \\
0.04\end{array}$ & $\begin{array}{r}16 \\
7\end{array}$ & $\begin{array}{l}4 \\
4\end{array}$ \\
\hline Max. 3 & $25710 / 9000$ & 0.39 & 19 & 58 oder 23800 & 0.43 & 13 & 19 \\
\hline Court/ZI. & 63 & & & 25 & & & \\
\hline Max. 1 & $27620 / 8702$ & 0.56 & 24 & 109 oder 28910 & 0.51 & 14 & 13 \\
\hline Max. 2 & $13251 / 5800$ & 0.05 & 16 & 161 oder 34112 & 0.16 & 9 & 29 \\
\hline Max. 3 & $19300 / 8003$ & 0.39 & 21 & 64 oder 24411 & 0.33 & 10 & 51 \\
\hline Undervelier-S/ZI. & 89 & & & 31 & & & \\
\hline Max. 1 & $12414 / 9012$ & 0.32 & 23 & 117 oder 29716 & 0.27 & 14 & 7 \\
\hline Max. 2 & $6614 / 8313$ & 0.34 & 19 & 79 oder 25904 & 0.44 & 9 & 13 \\
\hline Max. 3 & $19416 / 8313$ & 0.34 & 25 & 30 oder 21013 & 0.29 & 14 & 16 \\
\hline Undervelier- N/ZI. & 63 & & & 27 & & & \\
\hline Max. 1 & $19200 / 8100$ & 0.46 & 20 & 1 oder 18190 & $<0.01$ & 27 & 3 \\
\hline Max. 2 & $27915 / 8601$ & 0.49 & 18 & 121 oder 30102 & 0.36 & 13 & 22 \\
\hline Max. 3 & $33400 / 8300$ & 0.05 & 17 & 65 oder 24510 & 0.64 & 19 & 89 \\
\hline St-Sulpice/ZI. & 86 & & & 29 & & & \\
\hline Max. 1 & $13225 / 8822$ & 0.28 & 28 & 130 oder 31013 & 0.07 & 6 & 2 \\
\hline Max. 2 & $1016 / 8815$ & 0.30 & 23 & 179 oder 35906 & 0.48 & 9 & 11 \\
\hline Max. 3 & $5615 / 8512$ & 0.42 & 22 & 36 oder 21611 & 0.45 & 14 & 20 \\
\hline Combe Grède /ZI. & 42 & & & 18 & & & \\
\hline Max. 1 & $25001 / 8800$ & 0.56 & 22 & 60 oder 24006 & 1.00 & 15 & 10 \\
\hline Max. 2 & $16100 / 7800$ & 0.37 & 23 & 146 oder 32557 & 0.00 & 6 & 15 \\
\hline Max. 3 & $11011 / 7800$ & 0.07 & 6 & 86 oder 26690 & 0.00 & 4 & 24 \\
\hline Hauenstein/ZI. & 91 & & & 62 & & & \\
\hline Max. 1 & $2600 / 7109$ & 0.20 & 13 & 21 oder 20100 & 0.07 & 18 & 5 \\
\hline Max. 2 & $26013 / 8711$ & 0.38 & 24 & 64 oder 24401 & 0.43 & 11 & 16 \\
\hline Max. 3 & $33719 / 8914$ & 0.42 & 30 & 104 oder 28402 & 0.50 & 11 & 53 \\
\hline $\begin{array}{l}\text { Sonceboz/ZI. } \\
\text { Max } 1\end{array}$ & 85 & & & 21 & & & \\
\hline $\begin{array}{l}\text { Max. } 1 \\
\text { Max. } 2\end{array}$ & $9200 / 8700$ & 0.45 & 20 & 98 oder 27801 & 0.63 & 18 & 6 \\
\hline $\begin{array}{l}\text { Max. } 2 \\
\text { Max. } 3\end{array}$ & $\begin{array}{r}16706 / 9000 \\
5722 / 8015\end{array}$ & 0.28 & 23 & 147 oder 32702 & 0.37 & 6 & 20 \\
\hline $\begin{array}{l}\text { Max. } 3 \\
\text { Seyon/ZI. }\end{array}$ & & 0.27 & 21 & 34 oder 21490 & 0.00 & 4 & 23 \\
\hline Max. 1 & $12629 / 8722$ & 0.15 & 20 & 117 oder 29700 & 0.29 & 8 & 9 \\
\hline Max. 2 & $24006 / 8707$ & 0.64 & 17 & 78 oder 25801 & 0.64 & 13 & 18 \\
\hline Max. 3 & $34419 / 8616$ & 0.21 & 21 & 21 oder 20100 & 0.07 & 24 & 37 \\
\hline Covatanne/ZI. & 114 & & & 25 & & & \\
\hline Max. 1 & $23210 / 8210$ & 0.56 & 29 & 42 oder 22200 & 0.14 & 12 & 10 \\
\hline Max. 2 & $32711 / 8110$ & 0.39 & 23 & 135 oder 31516 & 0.08 & 6 & 12 \\
\hline Max. 3 & $11407 / 8307$ & 0.05 & 6 & 20 oder 20000 & 0.78 & 16 & 86 \\
\hline
\end{tabular}

An drei Stellen wurden in den Jurakalken Klüfte gemessen. Die gemessenen Kluftdaten wurden statistisch ausgewertet und die Resultate in der Tabelle 1 aufgelistet. Es zeigen sich zwei ganz prominente Kluftscharen; die dritte hat nur sehr wenig Gewicht. Ein Vergleich der Werte der Klusen von Undervelier-Nord und Undervelier-Süd ergibt, daß die beiden Sätze um etwa $30^{\circ}$ gegeneinander verschoben sind: ein weiteres Zeichen dafür, daß die beiden Klusen morphologisch und mechanisch nichts miteinander zu tun haben.
Im Vergleich mit den Talungsorientierungen wurde der Flußlauf wie üblich in 100-m-Schritten digitalisiert, und die Richtungen wurden einer statistischen Analyse unterworfen. Die Resultate sind im gegebenen Falle nicht sehr bestimmt. Trotzdem erkennt man, daß die der Karte zu entnehmende morphologische Haupttalrichtung mit der Polaren von 84 oder $264^{\circ}$ in zwei statistische Maxima bei 121 (301) und $65(245)^{\circ}$ aufgespalten ist.

Es ergibt sich eine gute $\left(3^{\circ}\right)$ Übereinstimmung zwischen den ersten Maxima bei Kluft- und Talorientierungen; 
jedoch ist das Gewicht des letzten sehr klein. Beim zweiten Maximum (Hauptrichtung) ist allerdings die Übereinstimmung nur leidlich $\left(22^{\circ}\right)$, wohl weil die Zahl der Flußsegmente für eine genauere Untersuchung nicht ausreicht. Im Hinblick auf die doch erkennbaren Übereinstimmungen muß wieder auf den gemeinsamen plattentektonischen Ursprung von Klus und Klüftung geschlossen werden.

\section{Klus von St-Sulpice}

In der asymmetrischen Klus von St-Sulpice (Abb. 3) liegt im Innern ein Boden, auf dem die mäandrierende Areuse zum Teil begradigt worden ist. Die den Boden begrenzenden Wände zeigen die Grenze zwischen Renggeri-Tonen und Terrain à Chailles. Am SE-Ende der Klus westlich von Fleurier treten Malmkalke zutage. Nach einem Tunnel am NW-Ende der Klus bietet sich ein Blick auf die gewaltige Karstquelle der Areuse, eine Source vauclusienne, tief unten, welche auch morphologisch andeutet, daß die "Klus» nicht von einem oberhalb nichtexistierenden Fluß (es gibt kein größeres Gewässer von Westen) hätte "eingesägt" werden können. Am oberen Ende der Schlucht stehen dickbankige Malmkalke an. An vier Aufschlüssen wurden Kluftorientierungen gemessen.

Morphologisch erscheint die Klus recht asymmetrisch: sie erweckt den Eindruck, durch eine dextrale Scherung mit einer E-W-Achse verzerrt worden zu sein (vgl. Abb. $3)$. Eine derartige Scherung würde gut dem neotektonischen europäischen Spannungsfeld mit NW-SE gerichteter P-Richtung entsprechen. Die Klus von St-Sulpice wurde auch in der Literatur als eine bereits aufgebrochene Doppelklus (aufgebrochenes Gewölbe; HANTKE, 1991, S. 262) bezeichnet.

Die Auswertung der gemessenen Kluftstellungen nach der üblichen Methode ist in der Tabelle 1 aufgeführt. Es bestehen offensichtlich zwei konjugierte Scharen, die eine bei $56^{\circ}$, die zweite bei $132^{\circ}$; eine dritte Schar liegt um $10^{\circ}$.

Anschließend wurden die Flußrichtungen in gewohnter Weise abgemessen und digitalisiert. Ein Problem war, daß von W kein Gewässer in die Klus einfließt; die Talungsrichtungen mußten daher auf der Westseite bis zur Source vauclusienne aus den Höhenkurven geschätzt werden. Später winden sich im Boden von St-Sulpice exogene Mäander, die zum Teil anthropogen begradigt wurden. Dabei wurde einfach die «blaue Linie» der Karte als Grundlage genommen, da die Statistik von selbst den Mittelwert bestimmt. Die Auswertung lieferte die in Tabelle 1 aufgelisteten Werte. Bei den drei Verteilungen findet eine Aufteilung in Scharen mit Polarenazimuten von 36,130 und $179^{\circ}$ statt.

Die Kluftscharen stimmen mit den entsprechenden Flußsegmentmaxima überein. Die Klüfte erscheinen im Uhrzeigersinn um $2-20^{\circ}$ gegenüber den Talungsmaxima verdreht. Dies würde dem Sinn der eben erwähnten dextralen Scherung entsprechen, so daß als Interpretation

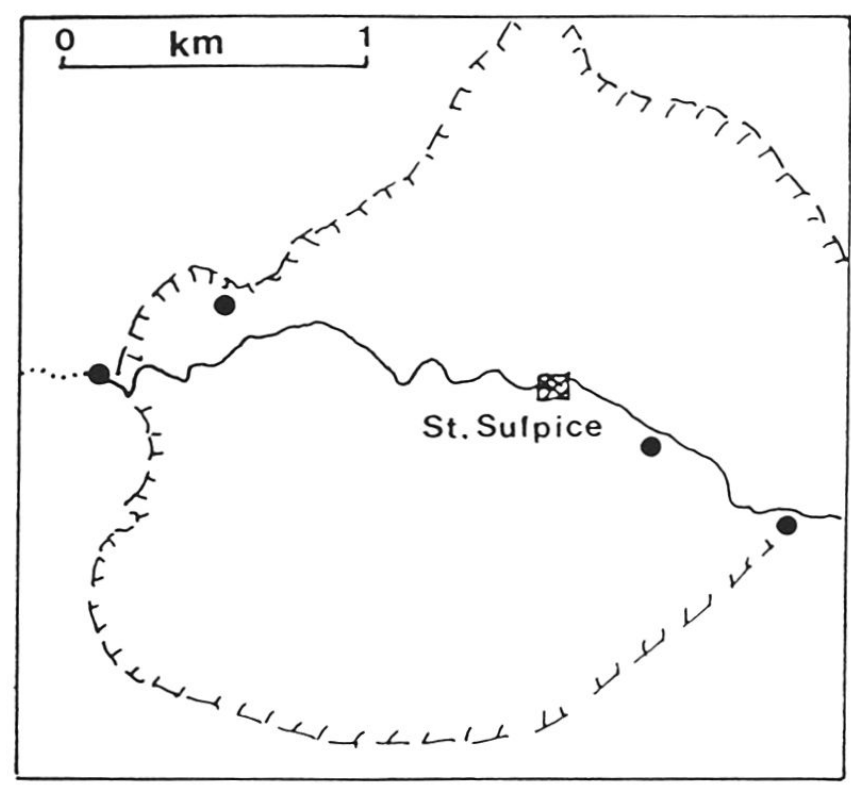

Abb. 3 Asymmetrische Klus: Plan der Klus von St-Sulpice mit den Kluftmeßstellen.

angenommen werden könnte, daß die Klüfte etwas älter wären als die rezente Scherung. Doch sind beide Formen durch die Tektonik entstanden.

\section{Combe Grède (Nordflanke des Chasseral)}

Als letzte klassische Klus wurde die Combe Grède untersucht. Dies ist eine ziemlich steil ansteigende Schlucht, die sich von Villeret aus mit einer Streichrichtung von S $23^{\circ} \mathrm{E}$ (Polare $\mathrm{N} 67^{\circ} \mathrm{E}$ ) in der Nordflanke des Chasseral liegt. Auf halber Höhe öffnet sie sich wie eine symmetrische Klus, ohne daß es sich dabei um einen «Durchbruch» handelt. Üblicherweise wird ihre Entstehung der Erosionswirkung des Wassers zugeschrieben, was schon rein morphologisch etwas weit hergeholt erscheint: Es fehlt das Einzugsgebiet für ein Gewässer der nötigen Größe.

An zwei Stellen wurden Kluftorientierungen gemessen. Es handelte sich in beiden Fällen um ein Jurakonglomerat. Die Auswertungen für drei vorausgesetzte Verteilungen sind in Tabelle 1 aufgelistet.

Wie üblich, galt es dann, die Kluftorientierungen mit den Talungsrichtungen zu vergleichen. Die Resulate der entsprechenden statistischen Untersuchung sind in Tabelle 1 aufgeführt. Dabei erscheint nur eine Verteilung sinnvoll; die anderen haben ein verschwindendes Gewicht. Die Klüftepolrichtung von $250(70)^{\circ}$ entspricht gut der Hauptpolarenrichtung (60 oder $240^{\circ}$ ) der Talung (Diskrepanz $10^{\circ}$ ). Dies zeigt, daß die Combe Grède wie die Klüfte durch tektonische Kräfte vorgezeichnet und nicht durch einen antezedenten Wasserlauf auserodiert wurde. 


\section{Übergänge}

\section{"Klus» Waldenburg-Langenbruck (Oberer Hauenstein BL)}

Als nächstes wurden «Klusen» betrachtet, die nicht eigentlich solche, sondern Übergänge durch/über einen Jurahöhenzug bilden. Als typisches Beispiel mag die «Klus» von Waldenburg bis Langenbruck(-Holderbank) dienen (Abb. 4), in der an vier Aufschlüssen Kluftstellungen eingemessen wurden. Bei all diesen - mit Ausnahme der südlichsten - wurde Hauptrogenstein (DoggerOolith, spätes Bajocien bis Bathonien) angetroffen, bei

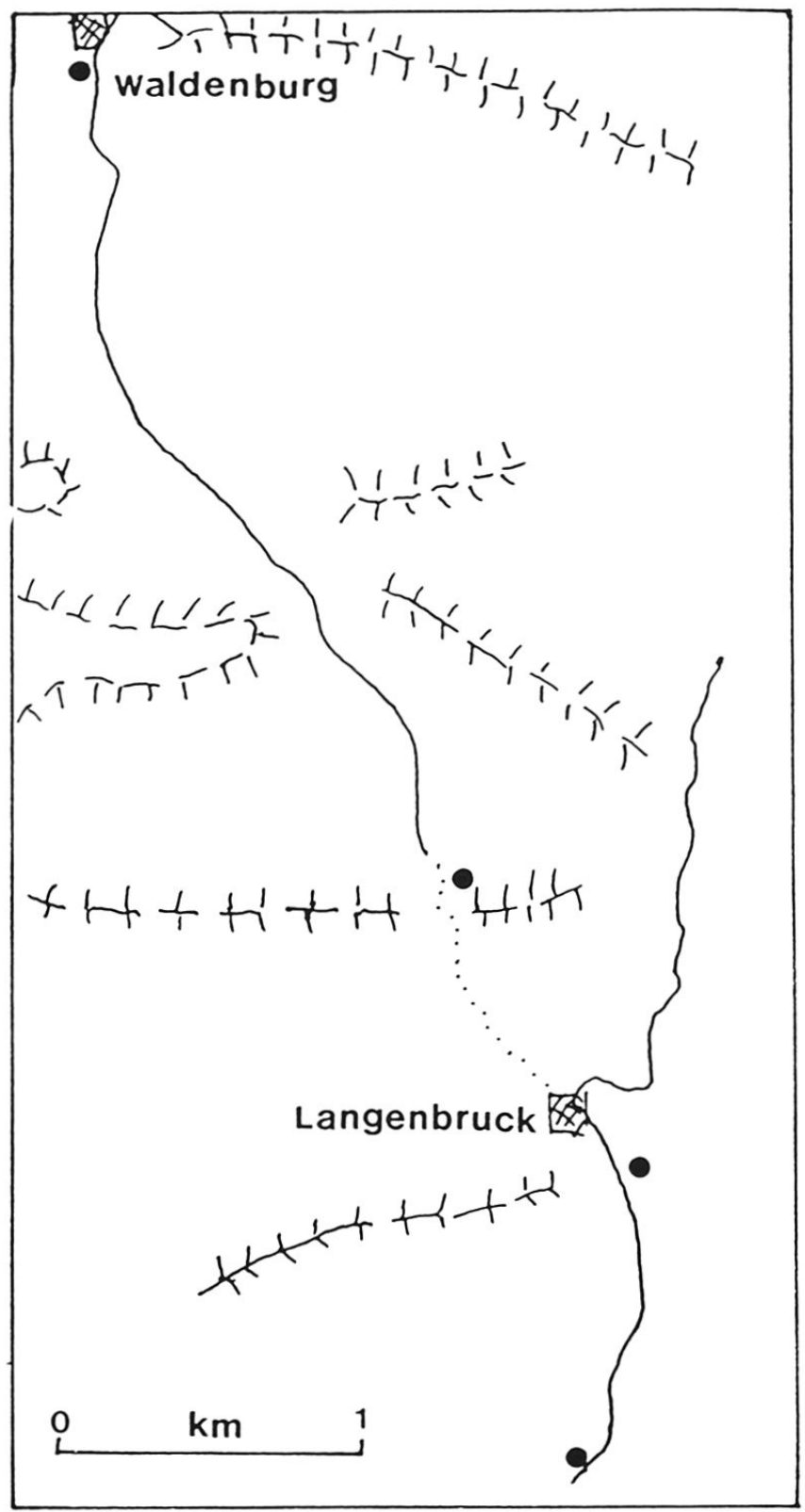

Abb. 4 Beispiel eines "Überganges": Lageplan der "Klus" von Waldenburg-Langenbruck (Oberer Hauenstein) mit den drei Kluftmeßstellen. der südlichsten Sequan, d. h. Oberes Oxfordien (Malm). Vom Oberen Hauenstein nach S zu steigt man in der Schichtfolge auf. Der Durchbruch bei Langenbruck erscheint nach visuellem Einvisieren sinistral versetzt. Bei der Strecke von Waldenburg bis S von Langenbruck handelt es sich offensichtlich nicht um eine einheitliche «Klus», sondern vielmehr um eine Sequenz von Durchbrüchen; dazwischen finden sich Mulden. Die Resultate der Auswertung der Kluftmessungen sind in Tabelle 1 aufgelistet.

Zur Untersuchung der Talungsrichtungen konnte im vorliegenden Falle nicht auf einen durchgehenden Flußlauf, sondern nur auf einige Äste, auf die Obere Frenke und den Schöntalbach, zurückgegriffen werden. Die entsprechenden Wasserläufe wurden in Abb. 4 ausgezogen. Die Läufe wurden wie gewohnt in Segmente digitalisiert und deren Richtungen statistisch ausgewertet. Die Resultate sind in Tabelle 1 aufgeführt.

Bei den Kluftauswertungen resultiert ein Maximum bei (Fallrichtung) etwa $260^{\circ}$ (Streichen $\mathrm{N} 10^{\circ} \mathrm{W}$ ). Das ist etwa die Richtung, die sich ergibt, wenn Anfang und Ende des Überganges miteinander verbunden werden. Diese Gerade entspricht auch dem Mittelwert des Zickzacks der Flußläufe. Zwei Maxima der Segmentverteilungen scharen sich um diesen; die dritte hat nur sehr kleines Gewicht. Sie entspricht damit der Haupttalungsrichtung, ein zweites Kluftmaximum steht mehr oder weniger $\left(54^{\circ}\right)$ konjugiert dazu. Wenn man die tektonische Druck(P-)Richtung als die Mittelnormale des kleineren Winkels (eben $54^{\circ}$ ) dieser zwei Kluftscharen nimmt, entspricht diese (140/23) der «europäischen" plattentektonischen Druckrichtung (NW-SE); die visuell festgestellte sinistrale Verschiebung paßt ebenfalls in das plattentektonische Spannungssystem.

\section{Klus von Sonceboz-Tavannes}

Die «Klus» von Sonceboz-Tavannes ist ein weiteres Beispiel eines «Überganges». An vier geeigneten Stellen wurden Kluftorientierungen gemessen und ein Vergleich mit den Talungsrichtungen angestellt. Beim Objekt handelt es sich um einen eigentlichen $P a \beta$, den Col de Pierre Pertuis, was schon von der Morphologie her jede Theorie, daß die "Klus» durch das «Einsägen» eines (antezedenten) Flusses enstanden sein könnte, falsifiziert.

Geologisch handelt es sich um feinkristalline, grau verwitternde Malmkalke; sie sind dick bis mittel gebankt mit etwas welliger Schichtung; darüber folgen gelblich anwitternde schiefrige Kalke. Nördlich des Passes befindet sich ein natürlicher Durchbruch, eben die Pierre pertuis (= Petra pertusa) durch eine Felsrippe.

Die Kluftdaten wurden wie üblich statistisch ausgewertet; die Resultate finden sich in Tabelle 1. Es zeigen sich zwei prominente Kluftscharen, die den üblicherweise in der Schweiz gefundenen plattentektonischen Kluftrichtungen (cf. SCHEIDEGGER, 1977) entsprechen.

Dann wurden die Talungsrichtungen untersucht. Da die «Klus» ein Paß ist, entwickelte sich nur auf der Südseite 
ein kleiner Bach. Auf der Nordseite tritt unterhalb des Überganges die Birs als Source de la Birse aus einer Karstquelle aus; nur wenig von diesem Flußlauf hat noch Bezug auf die "Klus». Doch wurden die erkennbaren Flußabschnitte gleichwohl auf beiden Seiten des Passes digitalisiert und der üblichen Analyse unterworfen. Die Resultate sind ebenfalls in Tabelle 1 aufgelistet. Es sind nur zwei der drei Verteilungen sinnvoll: die Haupttalung und eine um $50^{\circ}$ konjugierte dazu.

Die Haupttalung entspricht bei den drei Verteilungen ziemlich genau ( $6^{\circ}$ Diskrepanz) der Hauptkluftrichtung. Die übrigen Richtungen, sowohl bei den Klüften wie bei den Talsegmenten, sind von kleinem Gewicht und wohl eher zufällig. Jedenfalls lassen sie den Schluß zu, daß die Haupttalrichtung tektonischen Elementen entspricht und somit durch die Tektonik vorgezeichnet wurde.

\section{Randschwellenschluchten}

\section{Gorges du Seyon (Klus Valangin-Neuchâtel)}

Als nächstes betrachten wir Randschwellenschluchten. Als Paradigma dienen die Gorges du Seyon zwischen Valangin und Neuchâtel (Abb. 5), in ihnen wurden vier Aufschlüsse untersucht. Valangin ist die Typlokalität des "Valanginiens» (untere Kreide). Bei den Aufschlüssen handelt es sich um flach liegende Kalke, z. T. Konglomerate.

An den erwähnten vier Aufschlüssen wurden Kluftstellungen gemessen. Die Resultate ihrer numerischen Auswertungen finden sich in Tabelle 1. Es zeigt sich eine durchschlagende (Gewicht 0.64) Polrichtung (Maximum 2) mit Azimut $60^{\circ}=240^{\circ}$. Eine konjugierte dazu (Maximum 3 ) liegt bei $164^{\circ}=344^{\circ}$; die Mittelnormale des kleineren Winkels $\left(112^{\circ} / 5^{\circ}\right)$ entspricht der europäischen P-Richtung. Die dritte aufgelistete Richtung (Maximum 1) weicht nur um $39^{\circ}$ vom Maximum 3 ab und repräsentiert wohl «Abspaltungs»-Brüche. Dann wurden wie üblich die Talungsorientationen untersucht. Die Resultate der Auswertung sind in Tabelle 1 aufgeführt.

Die Werte der Kluftorientierungen wurden wieder mit der Orientationsstruktur der Talung verglichen. Eine direkte Verbindung von Eingang und Ausgang der Gorges du Seyon würde eine Polarenrichtung von $82^{\circ}$ ergeben, was um $22^{\circ}$ von der Hauptkluftrichtung (Maximum 2) abweicht. Es zeigt sich aber, daß in den Rechnungen mit drei Verteilungen die zwei ersten für Klüfte und Talungen recht gut zusammenstimmen. Man kann daher wieder denselben genetischen Ursprung für Klüfte und Talungen postulieren: beide sind plattentektonisch vorgezeichnet.

\section{Gorges de Covatanne südlich von Ste-Croix}

Als weiteres Beispiel einer Randschwellenschlucht wurden die Gorges de Covatanne untersucht. Morphologisch liegt der Ausgangspunkt der Untersuchung,

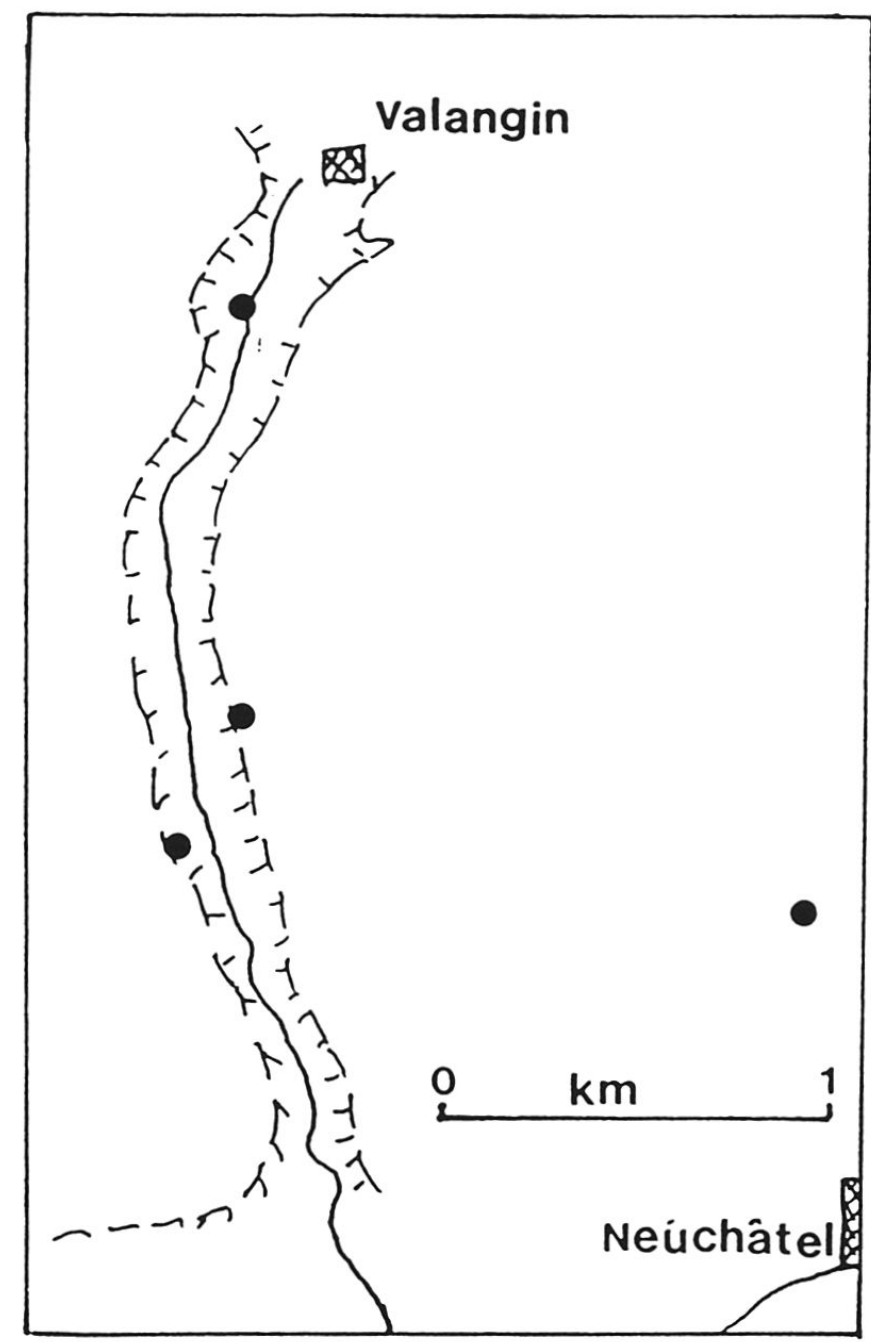

Abb. 5 Beispiel einer Randschwellenschlucht: Kartenskizze der Gorges du Seyon (Klus Neuchâtel-Valangin) mit den Kluftmeßstellen.

Ste-Croix, in einem Längstal zwischen zwei SW-NE streichenden Juraantiklinalen, wovon die südlichere von den Gorges de Covatanne gequert wird, die nach bisheriger Vorstellung von dem darin fließenden Arnon, einem bescheidenen Bach mit zwei Quellästen bei Ste-Croix, auserodiert worden wären. Das Niveau des Ausganges der Gorges liegt rund $450 \mathrm{~m}$ tiefer als der Eingang bei Ste-Croix. Weiter nach SE stellt sich eine flache Abdachung zum Neuenburgersee ein. Morphologisch fließt der Arnon aber keineswegs gerade in der Fallinie, sondern folgt im Zickzack auf erkennbaren Klüften, Spalten und Störungen. Engstellen wechseln mit «Böden». Geologisch handelt es sich bei dem Gestein um z. T. krümeligen Malmkalk; im unteren Teil der Schlucht findet sich ein junger Kalktuff, der in einem Tunnel durchbrochen wurde.

Kluftorientierungen wurden in der Schlucht an fünf Stellen gemessen. In Tabelle 1 finden sich die Resultate der entsprechenden statistischen Auswertungen. 
Entsprechend dem Plan der vorliegenden Arbeit wurden anschließend die Talungsrichtungen durch Digitalisierung des Flußlaufes bestimmt und statistisch ausgewertet; die Resultate sind auch in Tabelle 1 aufgelistet.

Ein Vergleich der Kluft- mit den Talungsrichtungen ergibt bei drei Verteilungen eine recht gute Übereinstimmung in den ersten beiden Maxima, die zueinander ziemlich genau konjugiert sind. Die aus diesen Kluftmaxima errechnete P-Richtung $\left(99^{\circ} / 12^{\circ}\right)$ entspricht wieder ungefähr der europäischen plattentektonischen Druckrichtung: Klüfte und Schlucht sind daher plattentektonisch vorgezeichnet.

\section{Schlußfolgerungen}

Die gängige These einer Genese der Juraklusen durch "Einsägen» von antezedenten Flüssen ist unhaltbar. Hierfür gibt es sechs Gründe:

1. Die Erosionskraft am Bett eines Flusses mit maximal $100 \mathrm{kPa}$ (MAGILligan, 1992) ist viel zu gering, als daß sich dieser hätte in festen Fels einsägen können.

2. In allen analysierten Fällen von Klusen sind ihre Flußsegmente nicht zufällig ( «random»), sondern systematisch orientiert. Nach den Aussagen des Antagonismusprinzips in der Landschaftsentwicklung bedeutet dies, daß die Genese der entsprechenden Formen nicht exogen, sondern endogen bedingt ist.

3. Normalerweise entspricht die Orientationsstruktur der Klusflußrichtungen jener der Kluftstellungen: wenigstens ein Kluftmaximum entspricht einem Flußrichtungsmaximum mit einer Diskrepanz von $\leqq 10^{\circ}$ (einzige Ausnahme: Court-Moutier $13^{\circ}$ ); das zweite mit einer Diskrepanz von $\leqq 20^{\circ}$ (einzige Ausnahme: UndervelierNord $22^{\circ}$ ).

4. Die Übereinstimmung von Fluß-und Kluftrichtungen in den Klusen legt nahe, daß beide durch analoge Prozesse gebildet wurden. Da bekannt ist, daß die Klüfte durch neoplattentektonische Vorgänge verursacht wurden, muß angenommen werden, daß auch Klusen durch solche Prozesse geschaffen wurden: sie folgen durch die rezente Plattentektonik vorgezeichneten Störungslinien.

5. Die Ausräumung der Klusen erfolgte parallel zu den Klüften. Dabei wurde der tektonisch angefallene sowie der mit dem Spaltenfrost längs den Klüften losgebrochene Schutt durch lösende und mechanische Wirkung des Wassers aus den engen Schluchten weggeführt. In den Kaltzeiten und in den kalten Jahreszeiten der Warmzeiten waren dies vor allem Schmelzwässer.

6. Über die ausgeräumte Quantität sind noch spezielle Studien nötig. Die hierfür zur Verfügung stehende Zeit erstreckt sich jedoch über einen weit längeren Zeitraum, als gemeinhin angenommen wird: sie beginnt mit der ersten Verformung, der ersten Faltenbildung, im Jura schon im mittleren Miozän, vor rund 15 Millionen Jahren.

\section{Literatur}

HANTKE, R. (1978): Eiszeitalter: Die jüngste Erdgeschichte der Schweiz und ihrer Nachbargebiete, Vol. 1. Thun: Ott, 468 pp. (reprint 1992 by ecomed, Landsberg/Lech).

HANTKE, R. (1991): Landschaftsgeschichte der Schweiz und ihrer Nachbargebiete. Thun: Ott, 309 pp.

KOHLBECK, F.K., SCHEIDEGGER, A.E. (1977): On the theory of joint orientation measurements. Rock Mechanics 9: 9-25.

LABHART, T.P. (1991): Geologie der Schweiz. Thun: Ott, $211 \mathrm{pp}$.

MAGILLIGAN, F. J. (1992): Thresholds and spacial variability of flood power during extreme floods. Geomorphology, 5: 373-390

SCHEIDEGGER, A. E. (1977): Kluftmessungen im Gelände und ihre Bedeutung für die Bestimmung des tektonischen Spannungsfeldes in der Schweiz. Geographica Helvetica, 32: $121-134$.

SCHEIDEGGER, A. E. (1979): The principle of antagonism in the Earth's evolution. Tectonophysics, 55: T7-T10.

SCHEIDEGGER, A.E. (1982): Principles of Geodynamics, 3d edition. Berlin: Springer, $395 \mathrm{pp}$.

SCHEIDEGGER, A.E. (1985): The significance of surface joints. Geophys. Surveys, 70: 259-271. 